\title{
Three-dimensional extended Newtonian (super)gravity
}

\author{
Nese Ozdemir, Mehmet Ozkan, Orhan Tunca and Utku Zorba \\ Department of Physics, Istanbul Technical University, \\ Maslak 34469 Istanbul, Turkey \\ E-mail: nozdemir@itu.edu.tr, ozkanmehm@itu.edu.tr, tuncaor@itu.edu.tr, \\ zorba@itu.edu.tr
}

ABSTRACT: We present a three dimensional non-relativistic model of gravity that is invariant under the central extension of the symmetry group that leaves the recently constructed Newtonian gravity action invariant. We show that the model arises from the contraction of a bi-metric model that is the sum of the Einstein gravity in Lorentzian and the Euclidean signatures. We also present the supersymmetric completion of this action which provides one of the very few examples of an action for non-relativistic supergravity.

Keywords: Classical Theories of Gravity, Supergravity Models, Extended Supersymmetry

ARXIV EPRINT: 1903.09377 
Newton-Cartan geometry has been studied extensively in recent years due to its use in condensed matter systems and non-AdS holography [1-11]. This geometric setup is the necessary framework to covariantize the Poisson equation of Newtonian gravity with the underlying symmetries are given by the centrally extended Galilei algebra, known as the Bargmann algebra [12-14]. While the Poisson equation was geometrized using NewtonCartan geometry long ago, an action principle for the Newtonian gravity has only recently been constructed [15]. The major feature of this action is that although the Bargmann algebra can capture the symmetries of the required field equation, it is not capable to obtain the Newtonian gravity action but an extension of that algebra with additional three new generators is necessary. It was, furthermore, mentioned in [15] that this new algebra can be obtained by a contraction of the direct sum of the Poincaré and Euclidean algebras in $D$ dimensions.

In this paper, we shall focus on the properties of the symmetry algebra that gives rise to Newtonian gravity action in three dimensions. The reason for that is twofold. First, three-dimensional non-relativistic geometries have found themselves direct applications in condensed matter physics $[2,3,16-18]$. Therefore, such new symmetries would presumably be expected to be relevant to a realistic theory. Second, gravity, both relativistically and non-relativistically, is technically much simpler to handle in three dimensions as it can be treated as a Chern-Simons gauge theory [19-24]. This technical advantage particularly allows us to immediately establish the three-dimensional action and to overcome two major difficulties with non-relativistic model construction, namely to obtain the model by taking a non-relativistic limit and to find the supersymmetric completion. In particular, we show that the three-dimensional model is the contraction of a bi-metric model that is the sum of the Einstein gravity in Lorentzian and the Euclidean signatures. Here, it is also worthwhile to briefly discuss the current status of non-relativistic supergravity. While supergravity in its relativistic formulation is now a well-established subject, its non-relativistic formulation is far from being complete with only very recent constructions [21, 25, 26]. Such models are particularly relevant to the construction of supersymmetric field theories on curved backgrounds by means of localization [27, 28]. Here, we provide the supersymmetric completion of the extended Newtonian gravity, which allows for such supersymmetric curved backgrounds, similar to extended Bargmann supergravity [21].

The spacetime symmetry algebra that is needed to construct an action principle for Newtonian gravity consists of the generators of the Bargmann algebra $\left\{H, P_{a}, G_{a}, J_{a b}, M\right\}$ as well as a set of additional generators $\left\{T_{a}, B_{a}, S_{a b}\right\}$ [15]. To construct a Chern-Simons action based on this algebra in three-dimensions, one needs to introduce a central extension in order to have a non-degenerate bilinear form. Here, we introduce two central charges, $Y$ and $Z$, and the non-zero commutation relations for the extended algebra are given by

$$
\begin{aligned}
& {\left[H, G_{a}\right]=-\epsilon_{a b} P^{b}, \quad\left[J, P_{a}\right]=-\epsilon_{a b} P^{b}, \quad\left[J, G_{a}\right]=-\epsilon_{a b} G^{b},} \\
& {\left[G_{a}, P_{b}\right]=\epsilon_{a b} M, \quad\left[G_{a}, G_{b}\right]=\epsilon_{a b} S, \quad\left[J, B_{a}\right]=-\epsilon_{a b} B^{b},} \\
& {\left[J, T_{a}\right]=-\epsilon_{a b} T^{b}, \quad\left[H, B_{a}\right]=-\epsilon_{a b} T^{b}, \quad\left[M, G_{a}\right]=-\epsilon_{a b} T^{b},} \\
& {\left[S, P_{a}\right]=-\epsilon_{a b} T^{b}, \quad\left[S, G_{a}\right]=-\epsilon_{a b} B^{b}, \quad\left[G_{a}, T_{b}\right]=-\epsilon_{a b} Y,} \\
& {\left[G_{a}, B_{b}\right]=\epsilon_{a b} Z, \quad\left[P_{a}, B_{b}\right]=-\epsilon_{a b} Y,}
\end{aligned}
$$


where $a=1,2$, and we used the three dimensional identities $J_{a b}=\epsilon_{a b} J$ and $S_{a b}=\epsilon_{a b} S$. This extended algebra can be equipped with an invariant non-degenerate bi-linear form

$$
\begin{aligned}
\left(P_{a}, B_{b}\right) & =\left(G_{a}, T_{b}\right)=\delta_{a b}, \\
(J, Y) & =-(M, S)=-(H, Z)=1,
\end{aligned}
$$

which we can use to construct a Chern-Simons action

$$
S=\frac{k}{4 \pi} \int \operatorname{Tr}\left(\mathrm{A} \wedge \mathrm{dA}+\frac{2}{3} \mathrm{~A} \wedge \mathrm{A} \wedge \mathrm{A}\right),
$$

where the gauge field $A=A_{\mu} d x^{\mu}$ is given by

$$
A_{\mu}=\tau_{\mu} H+e_{\mu}{ }^{a} P_{a}+\omega_{\mu} J+\omega_{\mu}{ }^{a} G_{a}+m_{\mu} M+s_{\mu} S+t_{\mu}{ }^{a} T_{a}+b_{\mu}{ }^{a} B_{a}+y_{\mu} Y+z_{\mu} Z .
$$

In its component form, this non-relativistic action is given by

$$
S_{\mathrm{ENG}}=\frac{k}{4 \pi} \int d^{3} x \varepsilon^{\mu \nu \rho}\left(e_{\mu}{ }^{a} R_{\nu \rho a}(B)+t_{\mu}^{a} R_{\nu \rho a}(G)+y_{\mu} R_{\nu \rho}(J)-m_{\mu} R_{\nu \rho}(S)-\tau_{\mu} R_{\nu \rho}(Z)\right),
$$

where we have used the curvatures

$$
\begin{aligned}
R_{\mu \nu}{ }^{a}(B) & =2 \partial_{[\mu} b_{\nu]}^{a}+2 \epsilon^{a b} \omega_{[\mu} b_{\nu] b}+2 \epsilon^{a b} s_{[\mu} \omega_{\nu] b}, \\
R_{\mu \nu}{ }^{a}(G) & =2 \partial_{[\mu} \omega_{\nu]}^{a}+2 \epsilon^{a b} \omega_{[\mu} \omega_{\nu] b}, \\
R_{\mu \nu}(J) & =2 \partial_{[\mu} \omega_{\nu]} \\
R_{\mu \nu}(S) & =2 \partial_{[\mu} s_{\nu]}+\epsilon_{a b} \omega_{[\mu}^{a} \omega_{\nu]}^{b} \\
R_{\mu \nu}(Z) & =2 \partial_{[\mu} z_{\nu]}+2 \epsilon^{a b} \omega_{[\mu a} b_{\nu] b} .
\end{aligned}
$$

We refer the action (5) as the extended Newtonian gravity. Although the extended Newtonian gravity is based on the central extension of the algebra that gives rise to an action for Newtonian gravity, it is quite distinct from the Newtonian gravity action of [15]. This can most easily be seen by the identity that connects the Riemann tensor to the group theoretical curvatures $R_{\mu \nu}(J)$ and $R_{\mu \nu}{ }^{a}(G)$

$$
R_{\rho \mu \nu}^{\sigma}=\epsilon^{a b} e^{\sigma}{ }_{a} e_{\rho b} R_{\mu \nu}(J)-\epsilon^{a b} e_{a}^{\sigma} \tau_{\rho} R_{\mu \nu b}(G) .
$$

This identity implies that the time component of the Ricci tensor is given by

$$
\tau^{\mu} \tau^{\nu} R^{\rho}{ }_{\mu \rho \nu}=-\epsilon^{a b} \tau^{\nu} e^{\mu}{ }_{a} R_{\mu \nu b}(G),
$$

which is precisely the leading term of the action for the Newtonian and extended Bargmann gravity [21-23] up to a redefinition $\omega^{a} \rightarrow \epsilon^{a b} \omega_{b}$. In three dimensions, this structure requires the invariant bi-linear form to have a non-zero $\left(P_{a}, G_{b}\right)$ component. On the other hand, such a non-zero component is forbidden by the algebra (1) as $S$ is not a central charge and most importantly it doesn't commute with $P_{a}$ and $G_{a}$. This implies that the non-relativistic models do not follow the relativistic analogy such that there is a unique two-derivative 
theory that admit Poincaré symmetries, namely the Einstein-Hilbert action, with both a metric and a Chern-Simons formulation. In non-relativistic setting, the symmetry algebra of the Newtonian gravity of [15] does not admit an invariant bilinear form without a central extension. Once the algebra is extended, the resulting Chern-Simons model is distinct from the Newtonian gravity action.

The extended Newtonian gravity is not the Chern-Simons formulation of the Newtonian gravity of [15] and yet it might be expected that it couples to matter in the same way that the Newtonian gravity couples to matter, i.e., its matter coupling gives rise to

$$
\tau^{\mu} \tau^{\nu} R_{\mu \rho \nu}^{\rho}=-\epsilon^{a b} \tau^{\nu} e^{\mu}{ }_{a} R_{\mu \nu b}(G) \propto j^{0},
$$

as an equation of motion which is the Poission equation for Newtonian gravity. In what follows we show the matter coupling of the extended Newtonian algebra resembles to that of extended Bargmann gravity [21] and admits backgrounds with non-trivial curvature whenever matter is present. When there is no matter coupling, the $z_{\mu}, b_{\mu}{ }^{a}$ and $s_{\mu}$ equations of motions for the extended Newtonian gravity (5) imply the standard curvature constraints

$$
R_{\mu \nu}(H)=0, \quad R_{\mu \nu}^{a}(P)=0, \quad R_{\mu \nu}(N)=0,
$$

where

$$
\begin{aligned}
R_{\mu \nu}(H) & =2 \partial_{[\mu} \tau_{\nu]}, \\
R_{\mu \nu}{ }^{a}(P) & =2 \partial_{[\mu} e_{\nu]}^{a}-2 \epsilon^{a b} \omega_{[\mu b} \tau_{\nu]}+2 \epsilon^{a b} \omega_{[\mu} e_{\nu] b}, \\
R_{\mu \nu}(N) & =2 \partial_{[\mu} m_{\nu]}+2 \epsilon^{a b} \omega_{[\mu a} e_{\nu] b} .
\end{aligned}
$$

Here, first equation implies that the spacetime can be foliated in an absolute time direction, while the last two equations identifies $\omega_{\mu}$ and $\omega_{\mu}{ }^{a}$ in terms of $\tau_{\mu}, e_{\mu}{ }^{a}$ and $m_{\mu}$. Hence the action (5) provides a model that is defined on torsionless Newton-Cartan geometry. When the matter coupling is included, however, any matter couplings to that includes $z_{\mu}$ would change the foliation constraint $R_{\mu \nu}(H)=0$. For simplicity let us consider the matter Lagrangian $\mathcal{L}_{m}$ to be independent of $z_{\mu}$ and consider the following action

$$
S=S_{\mathrm{ENG}}+\int d^{3} x e \mathcal{L}_{m} .
$$

We can look at the variation of the action with respect to $t_{\mu}{ }^{a}$ as it determines how the matter coupling would appear on the right-hand side of the Poisson equation via the identity (8). The $t_{\mu}{ }^{a}$ field equation read

$$
e^{-1} \varepsilon^{\mu \nu \rho} R_{\mu \nu a}(G)=-\frac{4 \pi}{k} T^{\rho}{ }_{a},
$$

where

$$
T^{\rho}{ }_{a}=e^{-1} \frac{\delta}{\delta t_{\rho}{ }^{a}}\left(e \mathcal{L}_{m}\right)
$$

This indicates that the time component of the Ricci tensor is given by

$$
\tau^{\mu} \tau^{\nu} R_{\mu \rho \nu}^{\rho} \propto e_{\mu}^{a} T_{a}^{\mu}
$$


The Newtonian gravity of [15] yields an equation for the Ricci tensor such that only the purely time-like component of the Ricci tensor is non-zero, i.e. $\tau^{\mu} \tau^{\nu} R^{\rho}{ }_{\mu \rho \nu} \propto j^{0}$. For the extended Newtonian gravity, the matter sources all components of the Riemann tensor similar to extended Bargmann gravity. Consequently, extended Newtonian gravity admits backgrounds with non-trivial curvature whenever matter is present.

Being a Chern-Simons theory, one of the immediate conclusions that can be made regarding the extended Newton gravity that it has no propagating degree of freedom. From a contraction viewpoint, this implies that the extended Newton gravity should be a non-relativistic limit of a non-interacting bi-metric gravity model due to its field content. This was noted in [15] to some extend that the $D$-dimensional analogue of the algebra (1) without the central extensions can be obtained from a contraction of the direct sum of the Poincare and Euclidean algebras in $D$ dimensions. The non-interacting model that we consider here is thus the sum of the Einstein gravity in Lorentzian and the Euclidean signatures plus a Chern-Simons action for two abelian gauge fields $Z_{1}$ and $Z_{2}$

$$
S=\frac{k \lambda}{4 \pi} \int \varepsilon^{\mu \nu \rho}\left(\eta_{A B} E_{1 \mu}^{A} R_{\nu \rho}^{B}\left(\Omega_{1}\right)+\delta_{A B} E_{2 \mu}^{A} R_{\nu \rho}^{B}\left(\Omega_{2}\right)+2 Z_{1 \mu} \partial_{\nu} Z_{2 \rho}\right)
$$

where $\eta_{A B}$ is Minkowski metric $\left(\eta_{A B}=\operatorname{diag}(-1,1,1)\right), \delta_{A B}$ is Euclidean metric $\left(\delta_{A B}=\right.$ $\operatorname{diag}(1,1,1))$ and $E_{\mu I}^{A}$ and $\Omega_{\mu I}^{A}$ with $I=1,2$ represent the dreibein and spin connections respectively. Here, the curvatures are given by

$$
\begin{aligned}
& R_{\nu \rho}^{A}\left(\Omega_{1}\right)=2 \partial_{[\mu} \Omega_{1 \nu]}^{A}+\epsilon^{A B C} \Omega_{1[\mu B} \Omega_{1 \nu] C}, \\
& R_{\nu \rho}^{A}\left(\Omega_{2}\right)=2 \partial_{[\mu} \Omega_{2 \nu]}^{A}+\tilde{\epsilon}^{A B C} \Omega_{2[\mu B} \Omega_{2 \nu] C},
\end{aligned}
$$

with the following convention for the Levi-Civita symmbols

$$
\varepsilon_{012}=-\varepsilon^{012}=-1, \quad \tilde{\varepsilon}_{012}=\tilde{\varepsilon}^{012}=1
$$

For the contraction procedure, we express the fields $E_{\mu I}^{A}, \Omega_{\mu I}^{A}, Z_{1 \mu}, Z_{2 \mu}$ as

$$
\begin{aligned}
E_{1 \mu}^{a} & =\lambda^{2} \sqrt{2} e_{\mu}{ }^{a}+\frac{\sqrt{2}}{2} t_{\mu}{ }^{a}, & E_{2 \mu}^{a} & =\lambda^{2} \sqrt{2} e_{\mu}{ }^{a}-\frac{\sqrt{2}}{2} t_{\mu}{ }^{a}, \\
\Omega_{1 \mu}^{a} & =\frac{1}{2 \sqrt{2} \lambda^{3}} b_{\mu}{ }^{a}+\frac{1}{\lambda} \omega_{\mu}{ }^{a}, & \Omega_{2 \mu}^{a} & =\frac{1}{2 \sqrt{2} \lambda^{3}} b_{\mu}{ }^{a}-\frac{1}{\lambda} \omega_{\mu}{ }^{a}, \\
E_{1 \mu}^{0} & =-\frac{1}{\lambda} y_{\mu}+2 \lambda^{3} \tau_{\mu}, & E_{1 \mu}^{0} & =-2 \lambda m_{\mu}+2 \lambda^{3} \tau_{\mu}, \\
\Omega_{1 \mu}^{0} & =\omega_{\mu}+\frac{1}{\lambda^{2}} s_{\mu}, & \Omega_{2 \mu}^{0} & =-\omega_{\mu}-\frac{1}{2 \lambda^{4}} z_{\mu}, \\
Z_{1 \mu} & =-\frac{1}{\lambda} m_{\mu}+2 \lambda \tau_{\mu} & Z_{2 \mu} & =s_{\mu}+2 \lambda^{2} \omega_{\mu} .
\end{aligned}
$$

Using these expressions in the action (15) and taking the limit $\lambda \rightarrow \infty$ we precisely recover the extended Newtonian gravity (5).

As mentioned, another advantage of the Chern-Simons formulation is that it allows us to find the supersymmetric description of the extended Newtonian gravity. To find the supersymmetric extension of the algebra (1) we proceed along the lines of [21] and consider 
five fermionic generators $Q^{ \pm}, W^{ \pm}$and $R$ that are all Majorana spinors. With the assistance of the computer algebra program Cadabra [29, 30], we find that the superalgebra has the following additional non-zero commutators

$$
\begin{array}{rlrl}
\left\{Q_{\alpha}^{+}, R_{\beta}\right\} & =\left(\gamma_{0} C^{-1}\right)_{\alpha \beta} M, & {\left[J, Q^{ \pm}\right]} & =-\frac{1}{2} \gamma_{0} Q^{ \pm}, \\
\left\{Q_{\alpha}^{+}, Q_{\beta}^{+}\right\} & =\left(\gamma_{0} C^{-1}\right)_{\alpha \beta} H, & {[J, R]} & =-\frac{1}{2} \gamma_{0} R, \\
\left\{Q_{\alpha}^{+}, Q_{\beta}^{-}\right\} & =-\left(\gamma_{a} C^{-1}\right)_{\alpha \beta} P^{a}, & {\left[J, W^{ \pm}\right]} & =-\frac{1}{2} \gamma_{0} W^{ \pm}, \\
\left\{Q_{\alpha}^{+}, W_{\beta}^{+}\right\} & =\left(\gamma_{0} C^{-1}\right)_{\alpha \beta} Y, & {\left[G_{a}, Q^{+}\right]} & =-\frac{1}{2} \gamma_{a} Q^{-}, \\
\left\{Q_{\alpha}^{+}, W_{\beta}^{-}\right\} & =\left(\gamma_{a} C^{-1}\right)_{\alpha \beta} T^{a}, & {\left[G_{a}, Q^{-}\right]} & =-\frac{1}{2} \gamma_{a} R, \\
\left\{Q_{\alpha}^{-}, Q_{\beta}^{-}\right\} & =\left(\gamma_{0} C^{-1}\right)_{\alpha \beta} M, & {\left[G_{a}, W^{-}\right]} & =-\frac{1}{2} \gamma_{a} W^{+}, \\
\left\{Q_{\alpha}^{-}, R_{\beta}\right\} & =-\left(\gamma_{a} C^{-1}\right)_{\alpha \beta} T^{a}, & =\frac{1}{2} \gamma_{a} W^{-}, \\
\left\{Q_{\alpha}^{-}, W_{\beta}^{-}\right\} & =\left(\gamma_{0} C^{-1}\right)_{\alpha \beta} Y, & =-\frac{1}{2} \gamma_{0} R, \\
\left\{R_{\alpha}, R_{\beta}\right\} & =-\left(\gamma_{0} C^{-1}\right)_{\alpha \beta} Y, & {\left[S, Q^{-}\right]} & =\frac{1}{2} \gamma_{0} W^{-}, \\
{\left[Z, Q^{+}\right]} & =\frac{1}{2} \gamma_{0} W^{+}, & {\left[B_{a}, Q^{ \pm}\right]} & =\frac{1}{2} \gamma_{a} W^{\mp}, \\
{[S, R]} & =\frac{1}{2} \gamma_{0} W^{+} . &
\end{array}
$$

Note that $Z$ is no longer a central charge in the supersymmetric extension of the algebra. The invariant supertrace is given by (2) extended with

$$
\begin{aligned}
\left(Q_{\alpha}^{+}, W_{\beta}^{+}\right) & =-2\left(C^{-1}\right)_{\alpha \beta}, \quad\left(Q_{\alpha}^{-}, W_{\beta}^{-}\right)=-2\left(C^{-1}\right)_{\alpha \beta}, \\
\left(R_{\alpha}, R_{\beta}\right) & =2\left(C^{-1}\right)_{\alpha \beta} .
\end{aligned}
$$

Introducing the gauge field

$$
\begin{aligned}
A_{\mu}= & \tau_{\mu} H+e_{\mu}{ }^{a} P_{a}+\omega_{\mu} J+\omega_{\mu}{ }^{a} G_{a}+m_{\mu} M+s_{\mu} S \\
& +t_{\mu}^{a} T_{a}+b_{\mu}{ }^{a} B_{a}+y_{\mu} Y+z_{\mu} Z+\bar{\psi}_{\mu}^{+} Q^{+} \\
& +\bar{\psi}_{\mu}^{-} Q^{-}+\bar{\rho}_{\mu} R+\bar{\phi}_{\mu}^{+} Q^{+}+\bar{\phi}_{\mu}^{-} Q^{-}
\end{aligned}
$$

the action for the extended Newtonian supergravity read

$$
\begin{aligned}
S= & \frac{k}{4 \pi} \int d^{3} x \varepsilon^{\mu \nu \rho}\left(e_{\mu}{ }^{a} R_{\nu \rho a}(B)+t_{\mu}{ }^{a} R_{\nu \rho a}(G)+y_{\mu} R_{\nu \rho}(J)-m_{\mu} R_{\nu \rho}(S)-\tau_{\mu} R_{\nu \rho}(Z)\right. \\
& \left.-\bar{\psi}_{\mu}^{+} R_{\nu \rho}\left(W^{+}\right)-\bar{\psi}_{\mu}^{+} R_{\nu \rho}\left(Q^{+}\right)-\bar{\phi}_{\mu}^{-} R_{\nu \rho}\left(W^{-}\right)-\bar{\phi}_{\mu}^{-} R_{\nu \rho}\left(W^{-}\right)+\bar{\rho}_{\mu} R_{\nu \rho}(R)\right)
\end{aligned}
$$


Here the supercovariant curvatures are given by

$$
\begin{aligned}
R_{\mu \nu}\left(Q^{+}\right) & =2 \partial_{[\mu} \psi_{\nu]}^{+}+\omega_{[\mu} \gamma_{0} \psi_{\nu]}^{+}, \\
R_{\mu \nu}\left(Q^{-}\right) & =2 \partial_{[\mu} \psi_{\nu]}^{-}+\omega_{[\mu} \gamma_{0} \psi_{\nu]}^{-}+\omega_{[\mu}^{a} \gamma_{a} \psi_{\nu]}^{+}, \\
R_{\mu \nu}(R) & =2 \partial_{[\mu} \rho_{\nu]}+\omega_{[\mu} \gamma_{0} \rho_{\nu]}+\omega_{[\mu}{ }^{a} \gamma_{a} \psi_{\nu]}^{-}+s_{[\mu} \gamma_{0} \psi_{\nu]}^{+}, \\
R_{\mu \nu}\left(W^{+}\right) & =2 \partial_{[\mu} \phi_{\nu]}^{+}+\omega_{[\mu} \gamma_{0} \phi_{\nu]}^{+}+\omega_{[\mu}^{a} \gamma_{a} \phi_{\nu]}^{-}-s_{[\mu} \gamma_{0} \rho_{\nu]}-z_{[\mu} \gamma_{0} \psi_{\nu]}^{+}-b_{[\mu}{ }^{a} \gamma_{a} \psi_{\nu]}^{-}, \\
R_{\mu \nu}\left(W^{-}\right) & =2 \partial_{[\mu} \phi_{\nu]}^{-}+\omega_{[\mu} \gamma_{0} \phi_{\nu]}^{-}-\omega_{[\mu}{ }^{a} \gamma_{a} \rho_{\nu]}-s_{[\mu} \gamma_{0} \psi_{\nu]}^{-}-b_{[\mu}{ }^{a} \gamma_{a} \psi_{\nu]}^{+} .
\end{aligned}
$$

These curvatures transform covariantly with respect to the supersymmetry transformation rules

$$
\begin{aligned}
\delta \tau_{\mu} & =-\bar{\epsilon}^{+} \gamma_{0} \psi_{\mu}^{+}, \\
\delta e_{\mu}{ }^{a} & =\bar{\epsilon}^{+} \gamma^{a} \psi_{\mu}^{-}+\bar{\epsilon}^{-} \gamma^{a} \psi_{\mu}^{+}, \\
\delta m_{\mu} & =-\bar{\epsilon}^{-} \gamma_{0} \psi_{\mu}^{-}-\bar{\epsilon}^{+} \gamma_{0} \rho_{\mu}-\bar{\eta} \gamma_{0} \psi_{\mu}^{+}, \\
\delta y_{\mu} & =-\bar{\epsilon}^{+} \gamma_{0} \phi_{\mu}^{+}-\bar{\chi}^{+} \gamma_{0} \pi_{\mu}^{+}-\bar{\epsilon}^{-} \gamma_{0} \phi_{\mu}^{-}-\bar{\chi}^{-} \gamma_{0} \psi_{\mu}^{-}+\bar{\eta} \gamma_{0} \rho_{\mu}, \\
\delta t_{\mu}^{a} & =-\bar{\epsilon}^{+} \gamma^{a} \phi_{\mu}^{-}-\bar{\chi}^{-} \gamma^{a} \psi_{\mu}^{+}+\bar{\epsilon}^{-} \gamma^{a} \rho_{\mu}+\bar{\eta} \gamma^{a} \psi_{\mu}^{-}, \\
\delta \pi_{\mu}^{+} & =\partial_{\mu} \epsilon^{+}+\frac{1}{2} \omega_{\mu} \gamma_{0} \epsilon^{+}, \\
\delta \pi_{\mu}^{-} & =\partial_{\mu} \epsilon^{-}+\frac{1}{2} \omega_{\mu} \gamma_{0} \epsilon^{-}+\frac{1}{2} \omega_{\mu}{ }^{a} \gamma_{a} \epsilon^{+}, \\
\delta \rho_{\mu} & =\partial_{\mu} \eta+\frac{1}{2} \omega_{\mu} \gamma_{0} \eta+\frac{1}{2} \omega_{\mu}{ }^{a} \gamma_{a} \epsilon^{-}+\frac{1}{2} s_{\mu} \gamma_{0} \epsilon^{+}, \\
\delta \phi_{\mu}^{+} & =\partial_{\mu} \chi^{+}+\frac{1}{2} \omega_{\mu} \gamma_{0} \chi^{+}+\frac{1}{2} \omega_{\mu}{ }^{a} \gamma_{a} \chi^{-}-\frac{1}{2} s_{\mu} \gamma_{0} \eta-\frac{1}{2} z_{\mu} \gamma_{0} \epsilon^{+}-\frac{1}{2} b_{\mu}{ }^{a} \gamma_{a} \epsilon^{-}, \\
\delta \phi_{\mu}^{-} & =\partial_{\mu} \chi^{-}+\frac{1}{2} \omega_{\mu} \gamma_{0} \chi^{-}-\frac{1}{2} \omega_{\mu}{ }^{a} \gamma_{a} \eta-\frac{1}{2} s_{\mu} \gamma_{0} \epsilon^{-}-\frac{1}{2} b_{\mu}{ }^{a} \gamma_{a} \epsilon^{+},
\end{aligned}
$$

where $\epsilon^{ \pm}, \chi^{ \pm}$and $\eta$ are the parameters of the local $Q^{ \pm}, W^{ \pm}$and $R$ transformations, respectively.

In this paper, we presented an action principle for a particular three-dimensional nonrelativistic gravity that is invariant under the central extension of the symmetry group that leaves the Newtonian gravity action invariant. The model is distinct from the Newtonian gravity both at the level of action and the matter coupling. By choosing fields appropriately, we show that this action can be obtained by a contraction procedure. Our model is of the Chern-Simons type which allowed us to establish the supersymmetric completion by extending the algebra with five supersymmetry generators.

The algebra that we present here can be extended to accommodate a cosmological constant. In this case, we expect that there are other invariant bi-linear forms, in particular, there might be an "exotic" extended Newtonian gravity that arises from the contraction of the sum of the "exotic" Einstein gravity in Lorentzian and the Euclidean signatures [19]. Furthermore, the algebra can also be extended to accommodate scale and Schrödinger symmetry along the lines of [23]. Once these extensions are established, it would also be interesting to see whether there is a further extension to include supersymmetry. 
The extended Newtonian gravity has no propagating degree of freedom as it is a ChernSimons gauge theory. Degree of freedom can be introduced by considering higher-derivative extensions or bi-gravity models. This can be most easily achieved by promoting our ChernSimons theory to a Chern-Simons-like theory, i.e., the action is still of the form (3) but $A_{\mu}^{I}$ is no longer has to be a gauge field [31]. While higher derivative models are expected to modify the Poisson equation for Newtonian gravity, the bi-gravity models are particularly relevant to a geometric interpretation of GMP states by providing a particular spin-2 planar Schrödinger equation [32-38]. Thus, it would very be interesting to see if one can construct non-relativistic interacting bi-gravity theories with the correct properties in light of the discussions that we provide here. Being a non-linear theory, it would also be interesting to develop a Hamiltonian formulation to analyze the degree of freedom of the resulting higher derivative or bi-gravity models in Chern-Simons like formalism.

Finally, the superalgebra that we presented in this paper is constructed by hand but obtained not from a contraction. In principle, this could be possible by considering the contraction of the sum of Poincaré and Euclidean supergravity along with an action for an abelian vector multiplet. The analysis of the matter multiplets, which can either be obtained by direct construction or by contraction, would be very interesting as they have important applications in supersymmetric non-relativistic field theories by means of localization.

\section{Acknowledgments}

We are greatful to Jan Rosseel for comments and suggestions. NO and UZ are supported in parts by Istanbul Technical University Research Fund under grant number TDK-201841133. The work of MO is supported in part by TUBITAK grant 118F091.

Open Access. This article is distributed under the terms of the Creative Commons Attribution License (CC-BY 4.0), which permits any use, distribution and reproduction in any medium, provided the original author(s) and source are credited.

\section{References}

[1] D.T. Son, Newton-Cartan Geometry and the Quantum Hall Effect, arXiv:1306.0638 [INSPIRE].

[2] A. Gromov and A.G. Abanov, Thermal Hall Effect and Geometry with Torsion, Phys. Rev. Lett. 114 (2015) 016802 [arXiv: 1407.2908] [INSPIRE].

[3] M. Geracie, D.T. Son, C. Wu and S.-F. Wu, Spacetime Symmetries of the Quantum Hall Effect, Phys. Rev. D 91 (2015) 045030 [arXiv:1407.1252] [InSPIRE].

[4] S. Moroz and C. Hoyos, Effective theory of two-dimensional chiral superfluids: gauge duality and Newton-Cartan formulation, Phys. Rev. B 91 (2015) 064508 [arXiv:1408.5911] [INSPIRE].

[5] C. Leiva and M.S. Plyushchay, Conformal symmetry of relativistic and nonrelativistic systems and AdS/CFT correspondence, Annals Phys. 307 (2003) 372 [hep-th/0301244] [INSPIRE]. 
[6] K. Balasubramanian and J. McGreevy, Gravity duals for non-relativistic CFTs, Phys. Rev. Lett. 101 (2008) 061601 [arXiv:0804.4053] [INSPIRE].

[7] D.T. Son, Toward an AdS/cold atoms correspondence: A Geometric realization of the Schrödinger symmetry, Phys. Rev. D 78 (2008) 046003 [arXiv: 0804.3972] [inSPIRE].

[8] C.P. Herzog, M. Rangamani and S.F. Ross, Heating up Galilean holography, JHEP 11 (2008) 080 [arXiv:0807.1099] [INSPIRE].

[9] C. Duval, M. Hassaine and P.A. Horvathy, The Geometry of Schrödinger symmetry in gravity background/non-relativistic CFT, Annals Phys. 324 (2009) 1158 [arXiv:0809.3128] [INSPIRE].

[10] S. Kachru, X. Liu and M. Mulligan, Gravity duals of Lifshitz-like fixed points, Phys. Rev. D 78 (2008) 106005 [arXiv:0808.1725] [INSPIRE].

[11] M. Taylor, Non-relativistic holography, arXiv:0812.0530 [INSPIRE].

[12] E. Cartan, Sur les variétés à connexion affine et la théorie de la relativité généralisée. (première partie), Annales Sci. Ecole Norm. Sup. 40 (1923) 325 [INSPIRE].

[13] E. Cartan, Sur les variétés à connexion affine et la théorie de la relativité généralisée. (première partie) (Suite), Annales Sci. Ecole Norm. Sup. 41 (1924) 1.

[14] A. Trautman, Sur la théorie newtonienne de la gravitation, Compt. Rend. Acad. Sci. Paris 247 (1963) 617.

[15] D. Hansen, J. Hartong and N.A. Obers, Action Principle for Newtonian Gravity, Phys. Rev. Lett. 122 (2019) 061106 [arXiv: 1807.04765] [INSPIRE].

[16] D.T. Son and M. Wingate, General coordinate invariance and conformal invariance in nonrelativistic physics: Unitary Fermi gas, Annals Phys. 321 (2006) 197 [cond-mat/0509786] [INSPIRE].

[17] C. Hoyos and D.T. Son, Hall Viscosity and Electromagnetic Response, Phys. Rev. Lett. 108 (2012) 066805 [arXiv:1109.2651] [INSPIRE].

[18] A.G. Abanov and A. Gromov, Electromagnetic and gravitational responses of two-dimensional noninteracting electrons in a background magnetic field, Phys. Rev. B 90 (2014) 014435 [arXiv:1401.3703] [INSPIRE].

[19] E. Witten, (2+1)-Dimensional Gravity as an Exactly Soluble System, Nucl. Phys. B 311 (1988) 46 [INSPIRE].

[20] A. Achucarro and P.K. Townsend, A Chern-Simons Action for Three-Dimensional anti-de Sitter Supergravity Theories, Phys. Lett. B 180 (1986) 89 [InSPIRE].

[21] E.A. Bergshoeff and J. Rosseel, Three-Dimensional Extended Bargmann Supergravity, Phys. Rev. Lett. 116 (2016) 251601 [arXiv: 1604.08042] [INSPIRE].

[22] G. Papageorgiou and B.J. Schroers, A Chern-Simons approach to Galilean quantum gravity in 2+1 dimensions, JHEP 11 (2009) 009 [arXiv:0907.2880] [INSPIRE].

[23] J. Hartong, Y. Lei and N.A. Obers, Nonrelativistic Chern-Simons theories and three-dimensional Hořava-Lifshitz gravity, Phys. Rev. D 94 (2016) 065027 [arXiv: 1604.08054] [INSPIRE].

[24] L. Avilés, E. Frodden, J. Gomis, D. Hidalgo and J. Zanelli, Non-Relativistic Maxwell Chern-Simons Gravity, JHEP 05 (2018) 047 [arXiv: 1802.08453] [INSPIRE]. 
[25] R. Andringa, E.A. Bergshoeff, J. Rosseel and E. Sezgin, 3D Newton-Cartan supergravity, Class. Quant. Grav. 30 (2013) 205005 [arXiv:1305.6737] [INSPIRE].

[26] E. Bergshoeff, J. Rosseel and T. Zojer, Newton-Cartan supergravity with torsion and Schrödinger supergravity, JHEP 11 (2015) 180 [arXiv:1509.04527] [INSPIRE].

[27] G. Festuccia and N. Seiberg, Rigid Supersymmetric Theories in Curved Superspace, JHEP 06 (2011) 114 [arXiv: 1105.0689] [INSPIRE].

[28] V. Pestun, Localization of gauge theory on a four-sphere and supersymmetric Wilson loops, Commun. Math. Phys. 313 (2012) 71 [arXiv:0712. 2824] [INSPIRE].

[29] K. Peeters, A Field-theory motivated approach to symbolic computer algebra, Comput. Phys. Commun. 176 (2007) 550 [cs/0608005] [INSPIRE].

[30] K. Peeters, Introducing Cadabra: A Symbolic computer algebra system for field theory problems, hep-th/0701238 [INSPIRE].

[31] E.A. Bergshoeff, O. Hohm, W. Merbis, A.J. Routh and P.K. Townsend, Chern-Simons-like Gravity Theories, Lect. Notes Phys. 892 (2015) 181 [arXiv: 1402.1688] [INSPIRE].

[32] S.M. Girvin, A.H. MacDonald and P.M. Platzman, Collective-Excitation Gap in the Fractional Quantum Hall Effect, Phys. Rev. Lett. 54 (1985) 581.

[33] S.M. Girvin, A.H. MacDonald and P.M. Platzman, Magneto-roton theory of collective excitations in the fractional quantum Hall effect, Phys. Rev. B 33 (1986) 2481 [INSPIRE].

[34] J. Maciejko, B. Hsu, S.A. Kivelson, Y. Park and S.L. Sondhi, Field theory of the quantum Hall nematic transition, Phys. Rev. B 88 (2013) 125137 [arXiv:1303.3041] [INSPIRE].

[35] Y. You, G.Y. Cho and E. Fradkin, Theory of Nematic Fractional Quantum Hall States, Phys. Rev. X 4 (2014) 041050 [arXiv: 1410.3390] [INSPIRE].

[36] F.D.M. Haldane, Geometrical Description of the Fractional Quantum Hall Effect, Phys. Rev. Lett. 107 (2011) 116801 [arXiv:1106.3375] [INSPIRE].

[37] A. Gromov and B. Bradlyn, Investigating Anisotropic Quantum Hall States with Bimetric Geometry, Phys. Rev. Lett. 119 (2017) 146602 [arXiv:1703.01304] [INSPIRE].

[38] A. Gromov and D.T. Son, Bimetric Theory of Fractional Quantum Hall States, Phys. Rev. X 7 (2017) 041032 [arXiv: 1705.06739] [INSPIRE]. 